\title{
The Catalytic Mitsunobu Reaction: A Critical Analysis of the Current State-of-the-Art
}

\author{
Rhydian H. Beddoe, ${ }^{a}$ Helen F. Sneddon ${ }^{b}$ and Ross M. Denton*a
}

The Mitsunobu reaction is widely regarded as the pre-eminent method for performing nucleophilic substitutions of alcohols with inversion of configuration. However, its applicability to large-scale synthesis is undermined by the fact that alcohol activation occurs at the expense of two stoichiometric reagents a phosphine and an azodicarboxylate. The ideal Mitsunobu reaction would be sub-stoichiometric in the phosphine and azodicarboxylate species and employ innocuous terminal oxidants and reductants to achieve recycling. This Review article provides a summary and analysis of recent advances towards the development of such catalytic Mitsunobu reactions.

\section{Introduction}

Bimolecular nucleophilic substitution $\left(\mathrm{S}_{\mathrm{N}} 2\right)$ reactions of alcohols are fundamental transformations in organic chemistry as they allow bond construction with inversion of stereochemistry. Ideally, such reactions would involve the direct substitution of a hydroxyl group with a nucleophile. Unfortunately, the poor leaving-group ability of the hydroxide anion and low electrophilicity of the hydroxyl-bearing carbon results in a substantial kinetic barrier for this hypothetical substitution process. In addition, nucleophiles that are also Brønsted basic may be able to deprotonate the alcohol preventing substitution. To overcome these issues, an alcohol must typically be converted to a more activated functionality, most commonly a halide or sulfonate ester, before nucleophilic substitution can occur. This requirement for an activating agent results in the generation of stoichiometric quantities of waste, which impacts negatively on the atom economy of these processes.

Stoichiometric phosphorus-mediated $\mathrm{S}_{\mathrm{N}} 2$ reactions of alcohols, are unique in that both activation and displacement steps occur in one pot. The most widely-used of these procedures is the Mitsunobu reaction, ${ }^{1}$ a "redox condensation", ${ }^{2}$ in which a hydroxyl-containing substrate, usually of a primary or secondary alcohol, is substituted with an acidic pro-nucleophile. ${ }^{3}$ The activation of the alcohol is achieved by reaction with a stoichiometric Morrison-Brunn-Huisgen betaine intermediate (1), which is formed by the in-situ reaction of triphenylphosphine and an azodicarboxylate reagent, generating the key alkoxyphosphonium salt (2) (Scheme 1). This intermediate is kinetically and thermodynamically unstable with respect to attack of the nucleophilic counter anion with inversion of stereochemistry. ${ }^{4}$ The Mitsunobu reaction exhibits excellent substrate scope, which encompasses a range of nitrogen, ${ }^{5}$ oxygen, ${ }^{1 a-c, 6}$ carbon, ${ }^{7}$ and sulfur ${ }^{8}$ pro-nucleophiles, and as such has become a vital toolbox reaction for synthetic chemists. However, the production of two by-products and the high energy nature of the azodicarboxylate reagent limits the use of the reaction on-scale and Mitsunobu couplings are often "designed out" of final synthesis routes to active pharmaceutical ingredients. ${ }^{9}$

In light of these well-documented issues, numerous creative strategies have been developed that allow chromatography-free purification of a crude Mitsunobu reaction mixture. ${ }^{10}$ Notable
Scheme 1. The Mitsunobu reaction, with key intermediates and commonly used pro-nucleophiles.

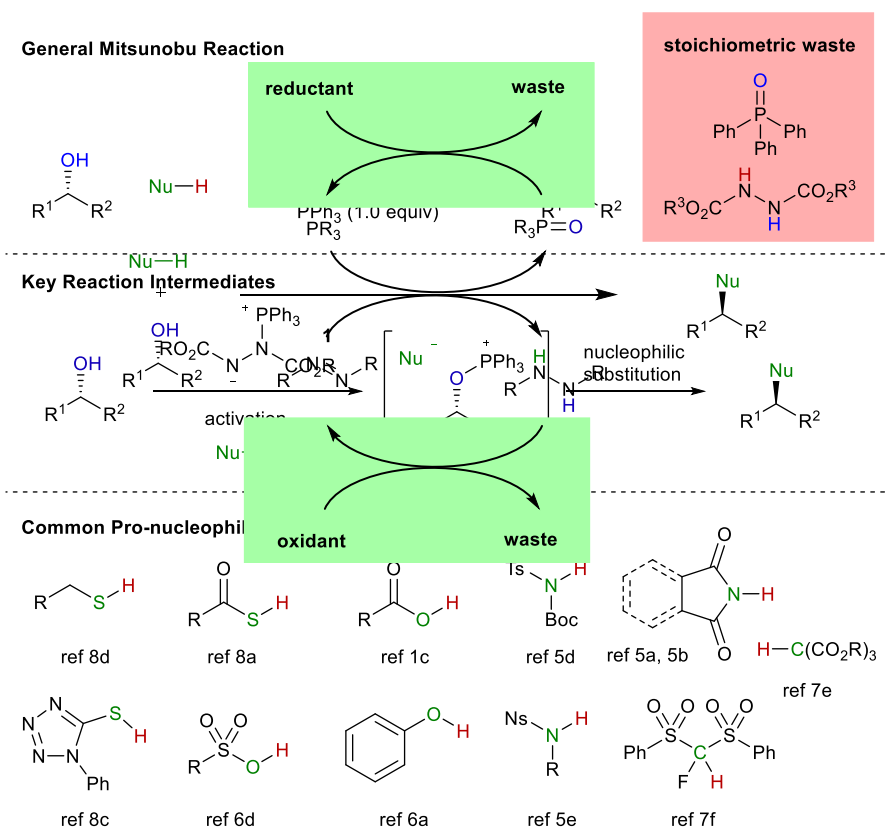

Scheme 2. A general strategy for a catalytic Mitsunobu reaction whereby the phosphine and azodicarboxylate are regenerated in-situ by reduction and oxidation respectively.

examples include Brønsted base/acid tagged reagents, ${ }^{11}$ polymer bound phosphine and azo species ${ }^{12}$ and reagents containing fluorous tags. ${ }^{13}$ In addition, Mitsunobu-active phosphorane reagents ${ }^{14}$ that facilitate azo-free Mitsunobu reactions have been identified, however, the broad pro-nucleophile scope has proved difficult to replicate using this approach.

More recent studies, seeking to tackle these problems, have sought to recycle the required stoichiometric reagents in-situ using milder, less hazardous terminal oxidants and reductants. This approach allows the phosphine or azodicarboxylate components to be used in sub-stoichiometric quantities (Scheme 2). However, the successful implementation of such reactions is challenging and centres on the identification of mutually compatible terminal oxidants and reductants that will affect chemoselective redox reactions. ${ }^{15}$ 
Herein, we present a summary of the recent progress that has been made towards the development of catalytic Mitsunobu reactions including metrics-based comparisons between the stoichiometric process and catalytic variants. ${ }^{16,17}$

\section{Azodicarboxylate Recycling}

The first example of a Mitsunobu protocol utilising a substoichiometric azodicarboxylate reagent, was reported by Toy in 2006. ${ }^{18}$ The redox strategy was predicated on the known oxidation of hydrazines using hypervalent iodine reagents. ${ }^{19}$ Toy's reaction (Scheme 3) made use of di(acetoxy)iodobenzene as the external,

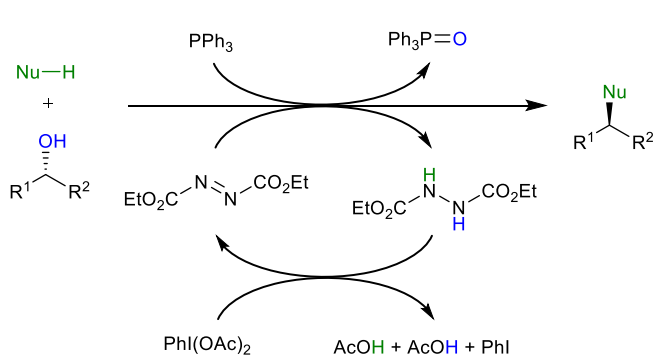

Scheme 3. Toy's azo-recycling strategy.

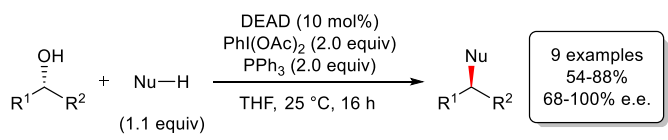

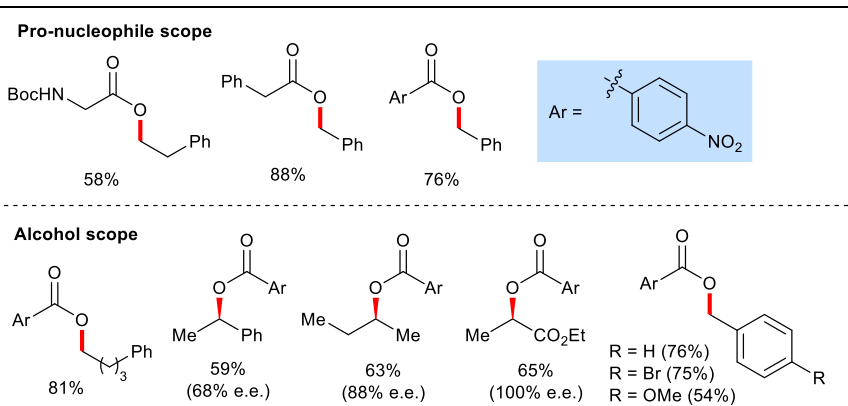

Scheme 4. The substrate scope of Toy's catalytic Mitsunobu reaction.

sacrificial oxidant. The typical hydrazine waste product associated with the Mitsunobu reaction was therefore replaced with iodobenzene and acetic acid; by-products which can be easily separated from a reaction mixture by distillation, rather than by chromatography.

A short optimization sequence, using 4-nitrobenzoic acid and 2phenylethanol as model substrates, revealed that $10 \mathrm{~mol} \%$ diethylazodicarboxylate (DEAD) could be used in combination with two equivalents of triphenylphosphine and di(acetoxy)iodobenzene to yield the first catalytic Mitsunobu system. The conditions were shown to tolerate both primary and secondary alcohols, with the latter proceeding with moderate-to-high levels of inversion (Scheme 4). It is noteworthy that a competing reaction with the acetic acid byproduct was observed, and this limited the range of compatible carboxylic acid pro-nucleophiles. An additional redox reaction involving the phosphine and di(acetoxy)iodobenzene results in a potentially Mitsunobu active dioxyphosphorane; however, the authors demonstrated that the background reaction in the absence of the diazodicarboxylate was not a significant pathway to the product. In a second publication, Toy and co-workers revisited the
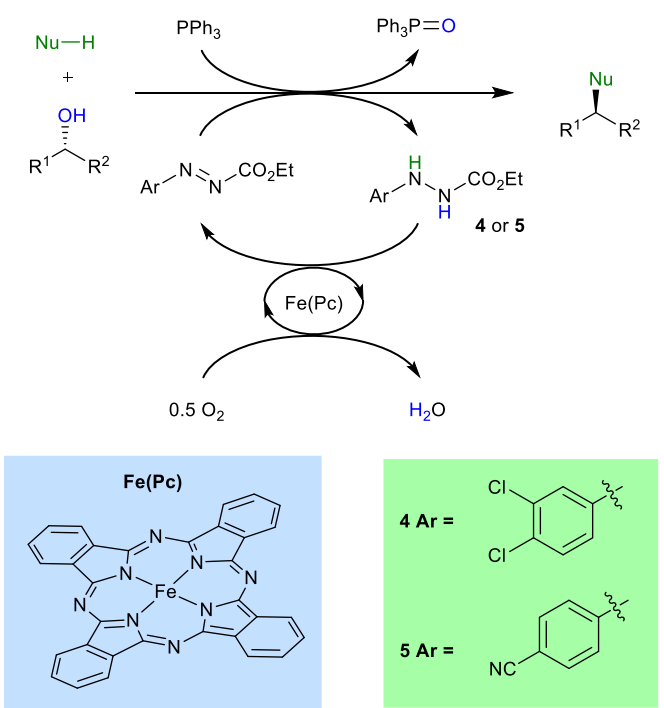

Scheme 5. Taniguchi's catalytic Mitsunobu reaction with oxidative recycling.
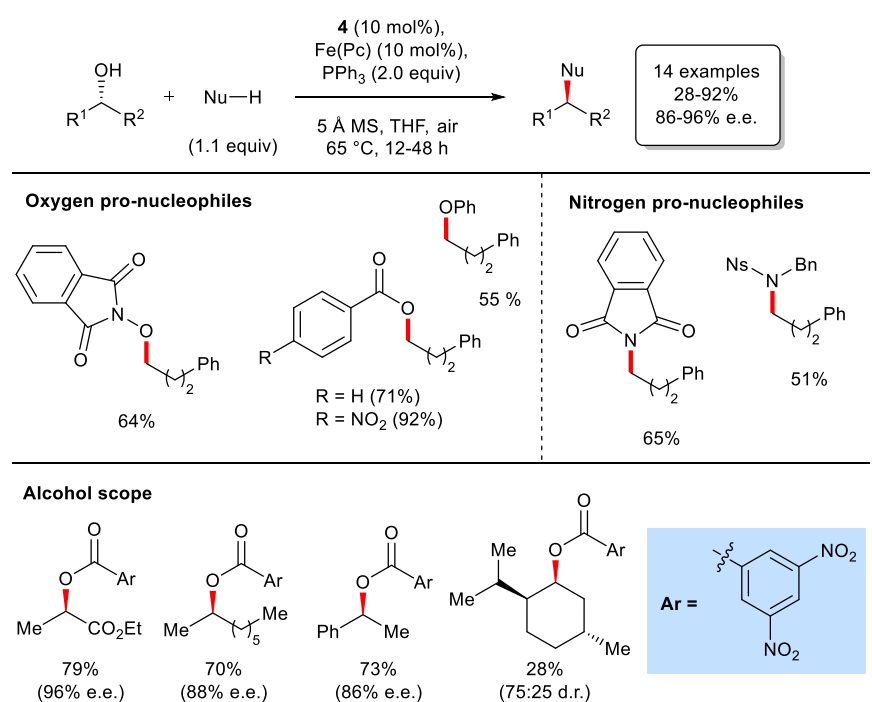

Scheme 6 . The substrate scope of Taniguchi's original catalytic Mitsunobu reaction.

catalytic reaction and developed an improved procedure in which 4nitro and 3,5-dinitrobenzoic acids were used as pronucleophiles. ${ }^{20}$ These studies represented a significant advance since they constituted the first fundamental change to the Mitsunobu protocol since 1967 and provided a stimulus for other research groups. 
Perhaps surprisingly following Toy's initial breakthrough this potentially rich area received no further attention until 2013 when Taniguchi and co-workers investigated an alternative oxidation system for the in-situ recycling of the azodicarboxylate reagent Building on their own studies on the aerobic oxidation of carbazates, ${ }^{21}$ they developed a working catalytic system to recycle a Mitsunobu-active arylazocarboxylate in-situ, using an iron

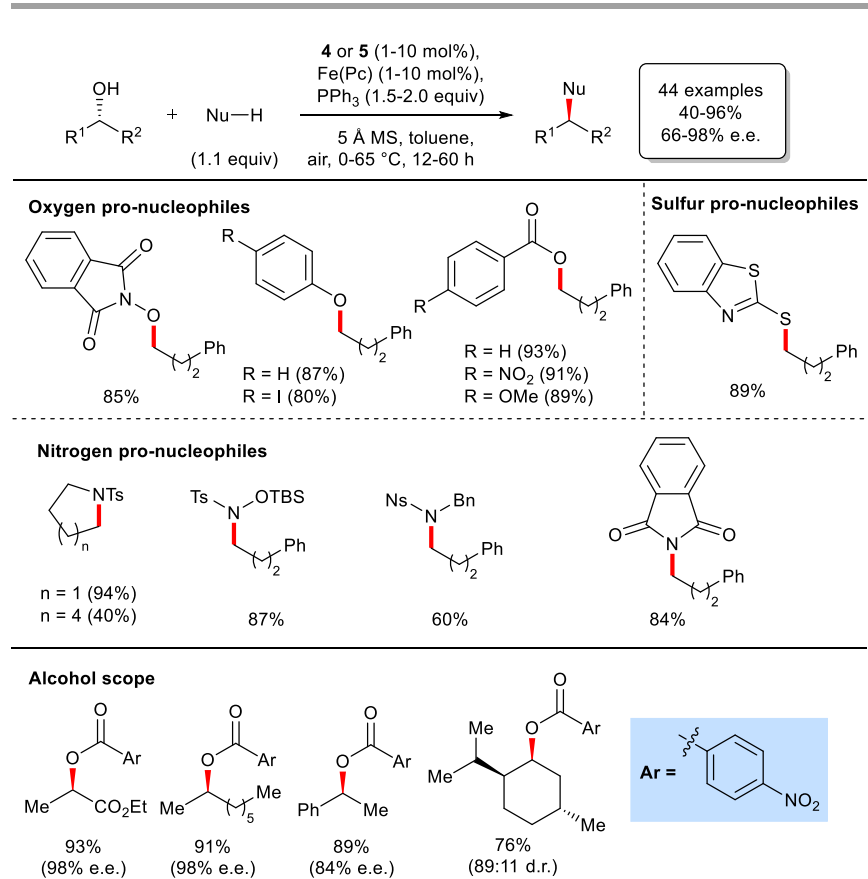

Scheme 7. The substrate scope of Taniguchi and Košmrlj's second-generation catalytic Mitsunobu reaction.

phthalocyanine co-catalyst, with oxygen as the terminal oxidant (Scheme 5). ${ }^{22}$ Arylhydrazinecarboxylates with electron deficient aryl groups, e.g. 4 and 5, were demonstrated to give the desired Mitsunobu activity, with 4 giving the best yields and levels of stereochemical inversion. ${ }^{23}$

In this system, the sole by-product of the oxidative recycling is water, which was removed from the reaction mixture using activated molecular sieves. Unlike the Toy procedure, the oxidant does not generate acetic acid as a competing pro-nucleophile and this allows a broader pro-nucleophile scope, which includes carboxylic acids, phenols, and activated amines. Generally, good yields were obtained with both primary and secondary alcohols and the expected inversion of stereochemistry was observed for substrates containing stereogenic centres (Scheme 6).

In a second comprehensive publication, Taniguchi, Košmrlj and co-workers carried out further optimisation, which included a Hammett study of the key elementary step involving the reaction between the arylhydrazinecarboxylate and phosphine, leading to the Morrison-Brunn-Huisgen betaine intermediate. ${ }^{24}$ The resulting second-generation catalytic reaction (Scheme 7) encompasses an expanded and impressive range of pronucleophiles, tolerates sterically hindered alcohols, affords excellent levels of stereochemical inversion and can be carried out under milder reaction conditions.

\section{Combined Azodicarboxylate and Phosphine Recycling: a "Fully Catalytic System"?}

The work described in the foregoing section highlights the advances that have been made in terms of removing a toxic and high

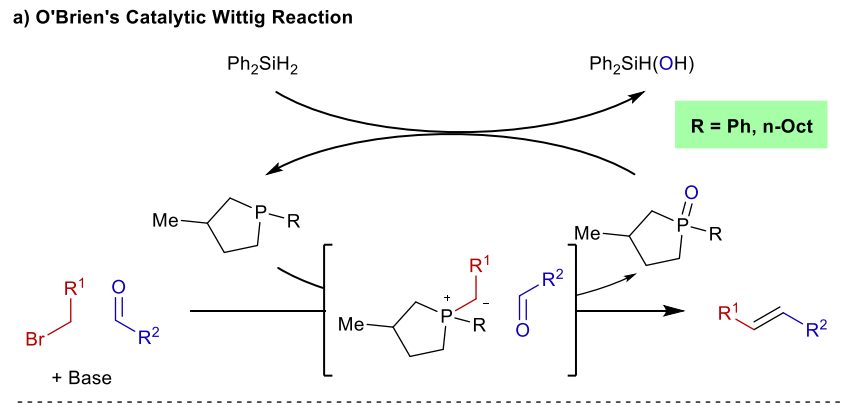

b) O'Brien/Aldrich's Catalytic Mitsunobu Reaction

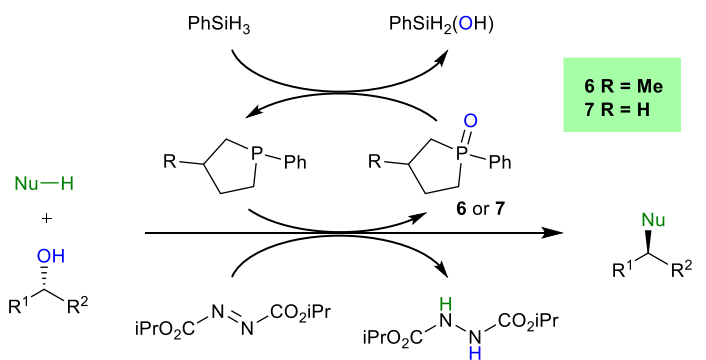

Scheme 8. a) O'Brien's design blueprint for a catalytic Wittig reaction and b) O’Brien and Aldrich's phosphine recycling system.

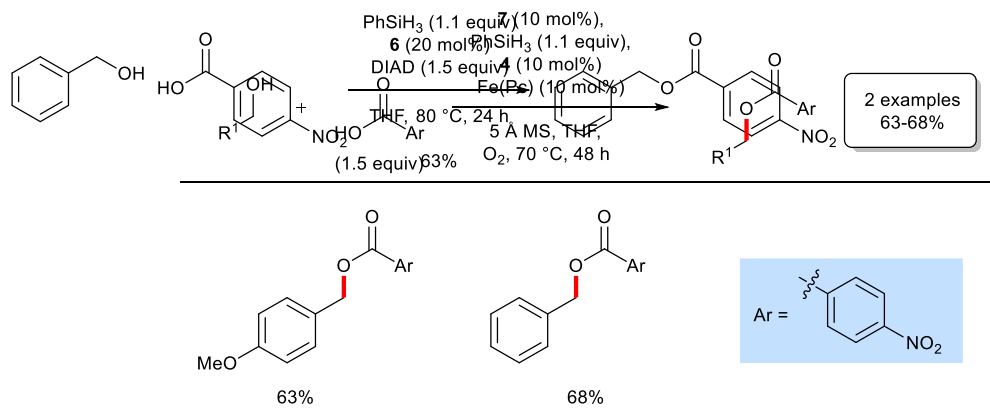

Scheme 9. The sole example of O'Brien's procedure, catalytic in phosphine. 

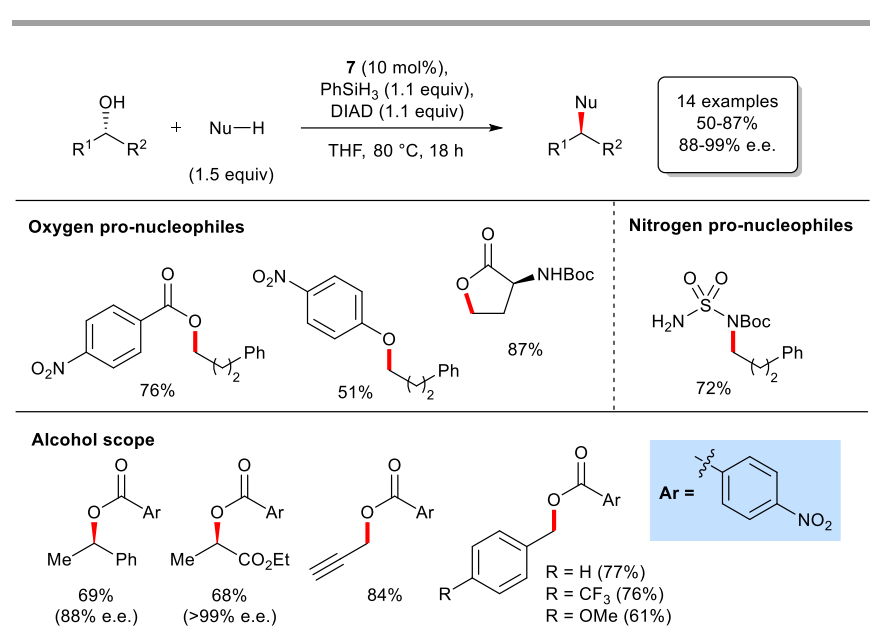

Scheme 10. Substrate scope for Aldrich's procedure.

energy stoichiometric oxidant from the Mitsunobu coupling. However, this progress notwithstanding, the Mitsunobu reaction is notorious for the production of phosphine oxide waste and the conspicuous challenge of a reaction catalytic in phosphine remained. Contemporaneous with the work of Toy and Taniguchi, a number of research groups were tackling this challenge in the broader context and reporting catalytic variants of other key phosphorous( $\mathrm{V}$ ) mediated transformations using silanes as chemoselective terminal reductants. ${ }^{25}$ The first example of this was a catalytic Wittig reaction developed by O'Brien and co-workers (Scheme 8a). ${ }^{26}$

Shortly after this key publication, $\mathrm{O}^{\prime}$ Brien applied this strategy to the Mitsunobu reaction and, in the patent literature, disclosed an esterification reaction in which substoichiometric phosphine oxide 6 (20 mol\%) was used as a putative precatalyst in combination with phenyl silane and stoichiometric diisopropylazodicarboxylate (Scheme 8b). ${ }^{27}$

While it is clear here that esterification has occurred in a

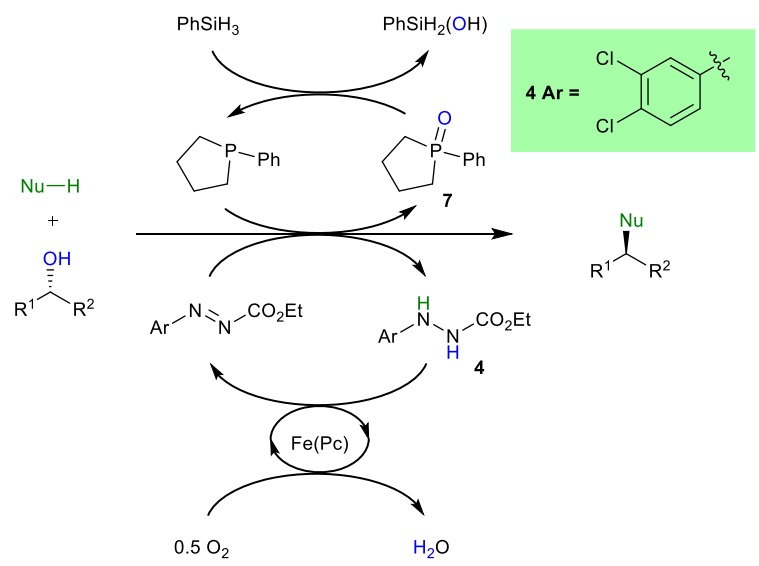

Scheme 11. The intended blueprint for Aldrich's 'fully catalytic' system, whereby both the azo and phosphine reagents are recycled.

Scheme 12. Substrate scope of Aldrich's 'fully catalytic' procedure.

reasonable yield, (Scheme 9) an inversion of configuration - a key attribute of the Mitsunobu esterification - was not demonstrated and, therefore, alternative esterification pathways (including silicon- mediated) cannot be ruled out. It should be noted that while the conventional Mitsunobu reaction has been the subject of detailed theoretical and mechanistic studies, ${ }^{4 p, 4 r, 4 s, 4 t}$ the catalytic variants described in this section have not been studied in detail (with the exception of Taniguchi and Košmrlj's system, Schemes 5 and 7.) Therefore, while the effect of variables such as pKa of the pronucleophile and steric demands of the alcohol are clear in the conventional system care must be taken when analysing the catalytic reactions.

This single but potentially important example was revisited by Aldrich and co-workers in 2015 and two further catalytic Mitsunobu protocols emerged, the first of which demonstrated phosphine recycling using the $\mathrm{O}^{\prime}$ Brien reductive approach. ${ }^{28,29}$ Using 4nitrobenzoic acid and benzyl alcohol as model substrates, the authors first established that the desired Mitsunobu coupling occurred in the presence of 10 mol\% phosphine oxide 6 , phenylsilane and stoichiometric quantities of diisopropyl azodicarboxylate (DIAD).

Scheme 13. a) A reinvestigation of the substrate scope of Aldrich's 'fully catalytic' system, carried out by the Taniguchi group; b) The key control experiment: the hydrazine is not required for product formation.

These occurred with comparable yields to a control experiment, which employed a traditional Mitsunobu protocol, using stoichiometric triphenylphosphine and DIAD reagents. The scope for this procedure was shown to tolerate both primary and secondary alcohols. Most importantly chiral non-racemic secondary alcohol

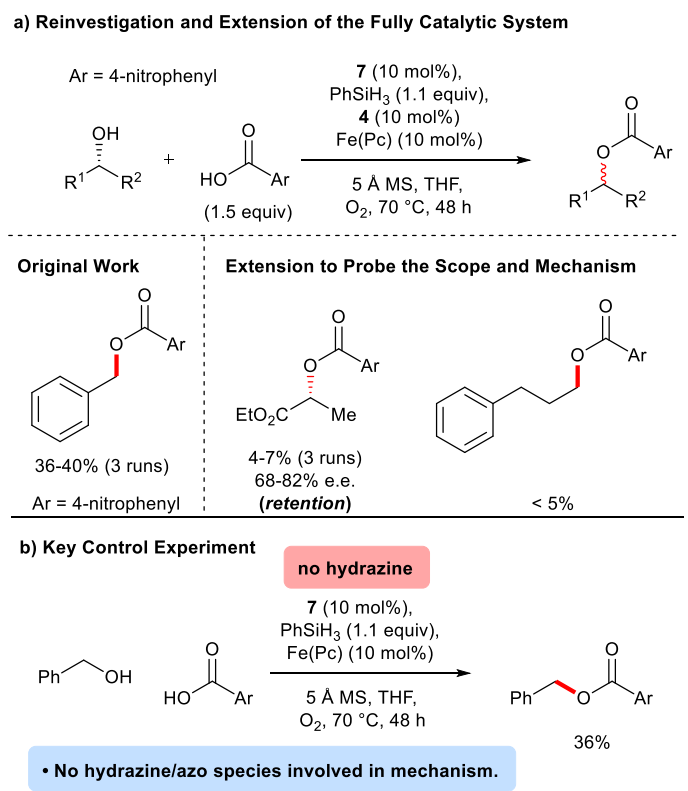

substrates gave products of high enantiomeric efficiency, with inversion of stereochemistry (Scheme 10).In a second study Aldrich and co-workers sought to integrate their phosphine recycling method with the Taniguchi iron- phthalocynanine azo recycling system to yield a 'fully catalytic system' (Scheme 11). ${ }^{28}$ The realisation of this strategy hinged on the compatibility of the silane reductant and the iron-phthalocyanine oxidation system, which would be expected to be highly challenging. ${ }^{29} \mathrm{~A}$ short optimisation 
sequence, using 4-methoxybenzyl alcohol and 4-nitrobenzoic acid as model substrates, revealed the requirement for $5 \AA$ A molecular sieves and an oxygen-enriched atmosphere to achieve acceptable yields. The substrate scope of this provocative double catalytic process was limited and, once again, did not include the inversion of a chiral nonracemic secondary alcohol.

This tantalizing possibility was investigated by Taniguchi, Košmrlj and co-workers ${ }^{30}$ in which the mechanism of the double catalytic system was probed. The study began by revisiting the esterification of benzyl alcohol (Scheme 13a). While the authors were able to obtain the product, the yield (36-40\% over three runs) was found to be inferior to the originally reported $68 \%$ (Scheme 12 ); however, the double catalytic system was qualitatively reproducible. A pivotal control experiment (Scheme 13b) was then preformed in the absence of the hydrazine. Surprisingly, the corresponding ester product was obtained in a very similar yield of $36 \%$, indicating that the hydrazine does not participate in the fully catalytic system and, therefore, the cycle depicted in Scheme 11 is not operational. Further important experiments were carried out in an attempt to expand the scope and provide additional mechanistic insight. An esterification of (-)-ethyl lactate afforded a very low $4-7 \%$ yield of product with $68-82 \%$ enantiomeric excess in favour of the retention product.

Finally, an esterification with 3-phenylpropan-1-ol also gave a very low yield of the corresponding ester. While these experiments do not eliminate the possibility of the doubly catalytic Mitsunobu reaction depicted in Scheme 11 being achieved, they clearly indicate that this ideal system is yet to be realized in practice. The successful implementation of such as system will depend upon the identification of mutually compatible terminal oxidants and reductants and remains an important challenge.

\section{Efficiency of the Catalytic Reactions}

A major deterrent to carrying out Mitsunobu reactions at scale is the waste generation and inherent inefficiency associated with the consumption of two stoichiometric reagents and the removal of the by-products so obtained. Since the Mitsunobu reaction is a "redox dehydration", a stoichiometric terminal reductant and oxidant will always be required for the catalytic Mitsunobu procedures discussed

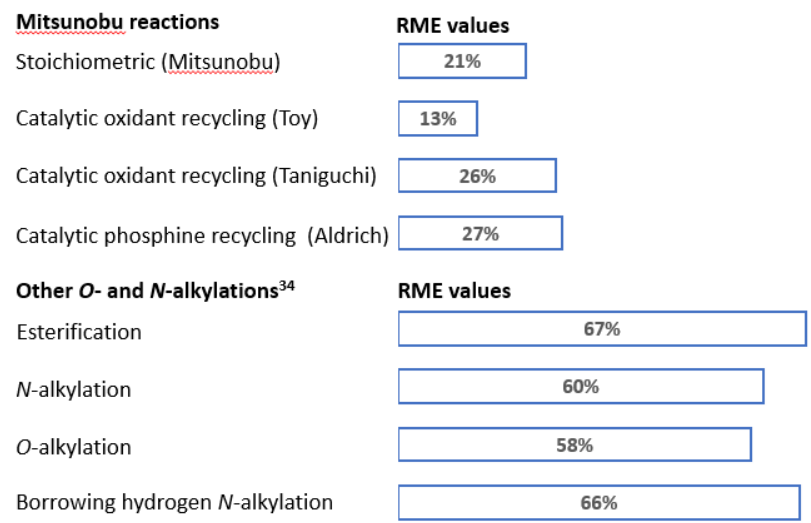

Figure 1. RME values for the stoichiometric Mitsunobu procedure, the Toy procedure (Scheme 3), the Taniguchi procedure (Scheme 5) and the Aldrich procedure (Scheme 8) contrasted with RME values for established esterification, $\mathrm{N}$-alkylation and $\mathrm{O}$-alkylation methods.

in this review. Therefore, any improvements in atom economy or waste generation are explicitly linked to the nature of the terminal oxidants and reductants employed. For example, although the Toy procedure allows for the Mitsunobu reaction to operate with substoichiometric quantities of DEAD, two equivalents of di(acetoxy)iodobenzene are required for re-oxidation, increasing the total amount waste generated. In contrast Taniguchi's procedure, in which oxygen is the terminal reductant, represents a more efficient protocol. Nevertheless, the process still requires superstoichiometric quantities of triphenylphosphine.

The reaction mass efficiency (RME) metric ${ }^{31}$ allows for a simple quantitative comparison of the newly developed methods (Figure 1). The RME value of a conventional stoichiometric Mitsunobu reaction (from 9 selected examples from ref. $2 a$ ) ${ }^{32}$ was calculated to be $21 \%$. In comparison with this benchmark figure the Toy procedure gave a reduced RME score (13\%) - a result of super-stoichiometric triphenylphosphine and terminal oxidant. Only modest improvements in RME scores were seen for both the Taniguchi procedure and Aldrich's method sub-stoichiometric exclusively in the phosphine reagent (Scheme 10) with RME scores of $26 \%$ and $27 \%$ respectively. ${ }^{33}$

Given that the Mitsunobu reaction is mainly used for esterification, the $\mathrm{O}$-alkylation of phenols and $\mathrm{N}$-alkylation the RME values of the catalytic reactions were compared with other established methods for these types of bond formation, which were calculated to be $67 \%, 60 \%$ and $58 \%$ respectively by Constable and coworkers. $^{34}$ Finally, an $\mathrm{N}$-alkylation reaction using the 'borrowing hydrogen' ${ }^{\prime 35,36}$ method was calculated to have a RME value of $66 \%$. The calculated values reveal a significant efficiency gap between all of the existing Mitsunobu protocols and the other competitive methods listed in the lower half of Figure 1. While some of this gap can be offset through the "added value" of the Mitsunobu reaction in terms of predictable stereochemical inversion and wide substrate scope, there is still much room for improvement and efficiency gain. Ultimately, such gains will come from the use of lower molecular weight redox reagents or, potentially, from alternative approaches to catalysis. 


\section{Conclusions}

The Mitsunobu reaction is widely used in academic and industrial laboratories and has, until recently, been used in its original form, which dates back to 1967 . Throughout this review we have highlighted the progress made towards the development of catalytic Mitsunobu reactions, beginning with the work of Toy. As this field continues to expand and new procedures emerge it is very likely that innovative reaction design will result in a series of Mitsunobu reactions whose efficiencies will exceed those of competitive methods and the true potential of this reaction will be realised for large-scale synthesis. ${ }^{9}$

\section{Conflicts of interest}

There are no conflicts of interest to declare.

\section{Acknowledgements}

We are grateful to the EPSRC (EP/L015633/1) and GlaxoSmithKline for funding.

\section{References}

1 For original publications, see (a) O. Mitsunobu, M. Yamada, T. Mukaiyama, Bull. Chem. Soc. Jpn. 1967, 40, 935-939; (b) O. Mitsunobu, M. Yamada, Bull. Chem. Soc. Jpn. 1967, 40, 23802382; (c) O. Mitsunobu, M. Eguchi, Bull. Chem. Soc. Jpn. 1971, 44, 3427-3430.

2 This term was coined by Mukaiyama to describe the use of an oxidant and a reductant simultaneously, to facilitate condensation reactions; T. Mukaiyama, Angew. Chem. Int. Ed. 1976, 15, 94-103.

3 For reviews, see (a) O. Mitsunobu, Synthesis 1981, 1-28; (b) B. R. Castro, Org. React. 1983, 29, 1-162; (c) D. L. Hughes, Org React. 1992, 42, 335-656; (d) D. L. Hughes, Org. Prep. Proced. 1996, 28, 127-164; (e) T. Y. S. But, P. H. Toy, Chem. Asian J. 2007, 2, 1340-1355; (f) K. C. K. Swamy, N. N. B. Kumar, E. Balaraman, K. V. P. P. Kumar, Chem. Rev. 2009, 109, 25512651; (g) S. Fletcher, Org. Chem. Front. 2015, 2, 739-752.

4 For further discussions of mechanism, see (a) D. C. Morrison, J. Org. Chem, 1958, 23, 1072-1074; (b) E. Brunn, R. Huisgen, Angew. Chem. Int. Ed. 1969, 8, 513-515; (c) E. Grochowski, B. D. Hilton, R. J. Kupper, C. J. Michejda, J. Am. Chem. Soc. 1982, 104, 6876-6877; (d) R. D. Guthrie, I. D. Jenkins, Aust. J. Chem. 1982, 35, 767-774; (e) M. von Itzstein, I. D. Jenkins, Aust. J. Chem. 1983, 36, 557-563; (f) W. Adam, N. Narita, Y. Nishizawa, J. Am. Chem. Soc. 1984, 106, 1843-1845; (g) M. von Itzstein, I. D. Jenkins, J. Chem. Soc. Perkin Trans. 1 1986, 437-445; (h) M. Varasi, K. A. M. Walker, M. L. Maddox, J. Org. Chem. 1987, 52 4235-4238; (i) D. L. Hughes, R. A. Reamer, J. J. Bergan, E. J. J. Grabowski, J. Am. Chem. Soc. 1988, 110, 6487-6491; (j) D. Crich, H. Dyker, R. J. Harris, J. Org. Chem. 1989, 54, 257-259; (k) A. Pautard-Cooper, S. A. Evans Jr., J. Org. Chem. 1989, 54, 2485-2488; (I) D. Camp, I. D. Jenkins, J. Org. Chem. 1989, 54, 3045-3049; (m) D. Camp, I. D. Jenkins, J. Org. Chem. 1989, 54, 3049-3054; (n) D. Camp, I. D. Jenkins, Aust. J. Chem. 1992, 45, 47-55; (o) S. R. Wilson, J. Perez, A. Pasternak, J. Am. Chem. Soc. 1993, 115, 1994-1997; (p) P. J. Harvey, M. von Itzstein, I. D. Jenkins, Tetrahedron 1997, 53, 3933; (q) T. Watanabe, I. D. Gridnev, T. Imamoto, Chirality 2000, 12, 346-351; (r) C. Ahn, R. Correia, P. DeShong, J. Org. Chem. 2002, 67, 1751-1753; (s)
J. McNulty, A. Capretta, V. Laritchev, J. Dyck, A. J. Robertson, Angew. Chem. Int. Ed. 2003, 42, 4051-4054; (t) S. Schenk, J. Weston, E. Anders, J. Am. Chem. Soc. 2005, 127, 12566-12576; (u) D. Camp, M. von Itzstein, I. D. Jenkins, Tetrahedron 2015, 71, 4946-4948; (u) D. Camp, P. J. Harvey, I. D. Jenkins, Tetrahedron 2015, 71, 3932-3938.

5 (a) O. Mitsunobu, M. Wada, T. Sano, J. Am. Chem. Soc. 1972, 94, 679-680; (b) M. Wada, T. Sano, O. Mitsunobu, Bull. Chem. Soc. Jpn. 1973, 46, 2833-2835; (c) J. Schweng, E. Zbiral, Justus Liebigs Ann. Chem. 1978, 1089-1095; (d) J. R. Henry, L. R. Marcin, M. C. Mclntosh, P. M. Scola, G. D. Harris, S. M. Weinreb, Tetrahedron Lett. 1989, 30, 5709-5712; (e) T. Fukuyama, C. K. Jow, M. Cheung, Tetrahedron Lett. 1995, 36, 6373-6374; (f) H. Huang, J. Y. Kang, J. Org. Chem. 2017, 82, 6604-6614.

6 (a) M. S. Manhas, W. H. Hoffman, B. Lal. A. K. Bose, J. Chem. Soc. Perkin Trans. 1 1975, 461-463; (b) I. D. Grice, P. J. Harvey, I. D. Jenkins, M. J Gallagher, M. G. Ranasinghe, Tetrahedron Lett. 1996, 37, 1087-1090; (c) N. G. Anderson, D. A. Lust, K. A. Colapret, J. H. Simpson, M. F. Malley, J. Z. Gougoutas, J. Org. Chem. 1996, 61, 7955-7958; (d) M. D. Pungente, L. Weiler, Org. Lett. 2001, 3, 643-646.

7 (a) M. Wada, O. Mitsunobu, Tetrahedron Lett. 1972, 13, 12791282; (b) T. Kurihara, M. Sugizaki, I. Kime, M. Wada, O. Mitsunobu, Bull. Chem. Soc. Jpn. 1981, 54, 2107-2112; (c) B. K. Wilk, Synth. Commun. 1993, 23, 2481-2484; (d) T. Tsunoda, C. Nagino, M. Oguri, S. Itô, Tetrahedron Lett. 1996, 37, 24592462; (e) M. C. Hillier, J-N. Desrosiers, J-F. Marcoux, E. J. J. Grabowski, Org. Lett. 2004, 6, 573-576; (f) G. K. S. Prakash, S. Chacko, S. Alconcel, T. Stewart, T. Matthew, G. A. Olah, Angew. Chem. Int. Ed. 2007, 46, 4933-4936.

8 (a) R. P. Volante, Tetrahedron Lett. 1981, 22, 3119-3122; (b) P. Rollin, Tetrahedron Lett. 1986, 27, 4169-4170; (c) T. Besson, M. A. Neirabeyeh, M-C. Viaud, P. Rollin, Synth. Commun. 1990, 20, 1631-1639; (d) J. R. Falck, J-Y. Lai, S-D. Cho, J. Yu, Tetrahedron Lett. 1999, 40, 2903-2906.

9 J. S. Carey, D. Laffan, C. Thomson, M. T. Williams, Org. Biomol. Chem. 2006, 4, 2337-2347.

10 For reviews, see (a) R. Dembinski, Eur. J. Org. Chem. 2004, 2763-2772; (b) S. Dandapani, D. P. Curran, Chem. Eur. J. 2004, 10, 3130-3138.

11 (a) D. Camp, I. D. Jenkins, Aust. J. Chem. 1988, 41, 1835-1839; (b) M. Kiankarimi, R. Lowe, J. R.McCarthy, J. P.Whitten, Tetrahedron Lett. 1999, 40, 4497-4500.

12 A. M. Harned, H. S. He, P. H. Toy, D. L. Flynn, P. R. Hanson, T. Uni, H. Kong, P. Road, J. Am. Chem. Soc. 2005, 127, 52-53.

13 (a) A. P. Dobbs, C. McGregor-Johnson, Tetrahedron Lett. 2002, 43, 2807-2810; (b) S. Dandapani, D. P. Curran, Tetrahedron, 2002, 58, 3855-3964.

14 (a) T. Tsunoda, F. Ozaki, S. Itô, Tetrahedron Lett. 1994, 35 , 5081-5082; (b) J. McNulty, A. Capretta, V. Laritchev, J. Dyck, A. J. Robertson, J. Org. Chem. 2003, 68, 1597-1600; (c) K. E. Elson, I. D. Jenkins, W. A. Loughlin, Org. Biomol. Chem. 2003, 1, 2958-2965.

15 It should be noted that the development of redox-neutral catalytic Mitsunobu reactions is an emerging field, however as the field is far less developed, it is beyond the scope of this review. (a) E. D. Nacsa, T. H. Lambert, Org. Lett. 2013, 15, 3841; (b) X. Tang, C. Chapman, M. Whiting, R. Denton, Chem. Commun. 2014, 50, 7340-7343.

16 For a review on catalytic bimolecular nucleophilic substitution reaction of alcohols, see J. An, R. M. Denton, T. H. Lambert, E. D. Nacsa, Org. Biomol. Chem. 2014, 12, 2993-3003.

17 A notable emission in this review is a recent study by Cibulka and co-workers on the Mitsunobu esterification of alcohols through photocatalytic recycling of the azo reagent. This procedure gave only ester products with retention of stereochemistry. Furthermore, the reaction gives appreciable 
yields in the absence of the azodicarboxylate reagent, implying that either there is a very strong occurrence of an azo-free background esterification, or the reaction does not follow a Mitsunobu mechanism. M. März, J. Chudoba, M. Kohout, R. Cibulka, Org. Biomol. Chem. 2017, 15, 1970-1975.

18 T. Y. S. But, P. H. Toy, J. Am. Chem. Soc. 2006, 128, 9636-9637.

19 R. M. Moriarty, I. Prakash, R. Penmasta, Synth. Commun. 1987, 17, 409-413.

20 T. Y. S. But, J. Lu, P. H. Toy, Synlett 2010, 8, 1115-1117.

21 T. Taniguchi, Y. Sugiura, H. Zaimoku, H. Ishibashi, Angew. Chem. Int. Ed. 2010, 49, 10154-10157.

22 D. Hirose, T. Taniguchi, H. Ishibashi, Angew. Chem. Int. Ed. 2013, 52, 4613-4617.

23 Taniguchi, Košmrlj and co-workers have since investigated the use of aryl-azocarboxylates as stoichiometric oxidants in the Mitsunobu reaction; D. Hirose, M. Gazvoda, J. Košmrlj, T. Taniguchi, J. Org. Chem. 2018, 83, 4712-4729.

24 D. Hirose, M. Gazvoda, J. Košmrlj, T. Taniguchi, Chem. Sci. 2016, 7, 5148-5159.

25 For reviews, see (a) H. A. van Kalkeren, F. L. van Delft, F. P. J. T. Rutjes, ChemSusChem 2013, 6, 1615-1624; (b) A. Voituriez, N. Saleh, Tetrahedron Lett. 2016, 57, 4443-4451; For selected highlights, see (c) H. A. van Kalkeren, S. H. A. M. Leenders, C. R. A. Hommersom, F. P. J. T. Rutjes, F. L. van Delft, Chem. Eur. J. 2011, 17, 11290-11295; (d) H. A. van Kalkeren, C. te Grotenhuis, F. S. Haasjes, C. A. Hommersom, F. P. J. T. Rutjes, F. L. van Delft, Eur. J. Org. Chem. 2013, 7059-7066; (e) H. A. van Kalkeren, J. J. Bruins, F. P. J. T. Rutjes, F. L. van Delft, $A d v$. Synth. Catal. 2012, 354, 1417-1421; (f) A. D. Kosal, E. E. Wilson, B. L. Ashfeld, Angew. Chem. Int. Ed. 2012, 51, 1203612040; (g) K. G. Andrews, R. M. Denton, Chem. Commun. 2017, 53, 7982-7985; (h) W. Zhao, P. K. Yan, A. T. Radosevich, J. Am. Chem. Soc. 2015, 137, 616-619; (i) T. V. Nykaza, T. S. Harrison, A. Ghosh, R. A. Putnik, A. T. Radosevich, J. Am. Chem. Soc. 2017, 139, 6839-6842; (j) T. V. Nykaza, A. Ramirez, T. S. Harrison, M. R. Luzung, A. T. Radosevich, J. Am. Chem. Soc. 2018, 140, 3103-3113.

26 (a) C. J. O'Brien, J. L. Tellez, Z. S. Nixon, L. J. Kang, A. L. Carter, S. R. Kunkel, K. C. Przeworski, G. A. Chass, Angew. Chem. Int. Ed. 2009, 48, 6836-6839; (b) C. J. O'Brien, F. Lavigne, E. E. Coyle, A. J. Holohan, B. J. Doonan, Chem. Eur. J. 2013, 19, 5854-5858; (c) C. J. O'Brien, Z. S. Nixon, A. J. Holohan, S. R. Kunkel, J. L. Tellez, B. J. Doonan, E. E. Coyle, F. Lavigne, L. J. Kang, K. C. Przeworski, Chem. Eur. J. 2013, 19, 15281-15289; (d) E. E. Coyle, B. J. Doonan, A. J. Holohan, K. A. Walsh, F. Lavigne, E. H. Krenske, C. J. O'Brien, Angew. Chem. Int. Ed. 2014, 53, 12907-12911.

27 C. J. O'Brien (Univ. Texas, USA) WO 2010/118042 A3, 2010.

28 J. A. Buonomo, C. C. Aldrich, Angew. Chem. Int. Ed. 2015, 54, 13041-13044.

29 S. Davey, Nat. Chem. 2013, 5, 358.

30 D. Hirose, M. Gazvoda, J. Košmrlj, T. Taniguchi, Org. Lett. 2016 18, 4036-4039.

31 A. D. Curzons, N. D. Mortimer, D. J. C. Constable, V. L. Cunningham, Green Chem. 2001, 3, 1-6.

32 The following eaxmples were selected as representative; Table 1, entries 4, 5 and 6; Table 5, entries 4, 5 and 7; Table 6, entries 4,5 and 6 .

33 Due to the issues associated with its scope and reproducibility we have omitted Aldrich's fully catalytic system from our analyses.

34 D. J. C Constable, A. D. Curzons, V. L. Cunningham, Green Chem. 2002, 4, 521-527.

35 For reviews, see (a) M. H. S. A Hamid, P. A. Slatford, J. M. J. Williams, Adv. Synth. Catal. 2007, 349, 1555-1575; (b) A. J. A Watson, J. M. J. Williams, Science, 2010, 329, 635-636; (c) G. E. Dobereiner, R. H. Crabtree, Chem. Rev. 2010, 110, 681-703. (d) G. Guillena, D. J. Ramón, M. Yus, Chem. Rev. 2010, 110,
1611-1641; (e) C. Gunanathan, D. Milstein, Science, 2013, 341, 249; (f) Q. Yang, Q. Wanga, Z. Yu, Chem. Soc. Rev. 2015, 44 2305-2329. For seminal publications, see (g) M. H. S. A. Hamid, C. L. Allen, G. W. Lamb, A. C. Maxwell, H. C. Maytum, A. J. A Watson, J. M. J. Williams, J. Am. Chem. Soc. 2009, 131, 1766; (h) T. Yan, B. L. Feringa, K. Barta, Nat. Commun. 2014, 5, 5602; (i) S. Elangovan, J. Neumann, J-B. Sortais, K. Junge, C. Darcel, M. Beller, Nat. Commun. 2016, 7, 12641.

36 The mean RME value was calculated from the examples in table 3 from publication $35 \mathrm{~g}$.

\section{Author Profiles}

Rhydian Beddoe graduated from the University of Bristol in 2016 where he conducted a final year project with Professor Kevin Booker-Milburn investigating the photochemical generation of drug scaffolds. He is currently studying towards his PhD under the supervision of Dr. Ross Denton, as part of the EPSRC funded Centre for Doctoral Training in Sustainable Chemistry, at the University of Nottingham.

Helen Sneddon obtained her PhD in 2005 from the University of Cambridge under the supervision of Professor Steven Ley. Following postdoctoral studies at the University of California, Irvine with Professor Larry Overman, she joined GlaxoSmithKline in 2007, and where in 2011 she founded a Green Chemistry group which she still leads.

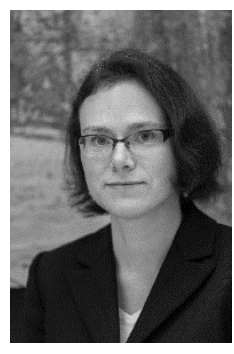

Ross Denton obtained his PhD in 2005 from The University of Nottingham under the supervision of Professor Jim Anderson. Following postdoctoral studies at The Scripps Research Institute with Professor K.C. Nicolaou and Cambridge with Professor Steven Ley, he returned to Nottingham to begin his independent career in 2008. His research

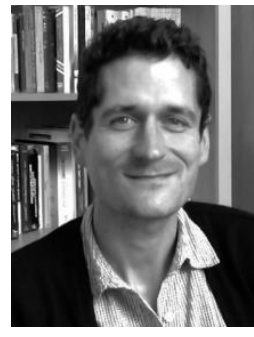
involves the development of new synthesis methods, new catalytic reactions and their application in target synthesis. 